\title{
Uplift of an elastic membrane by a viscous flow
}

\author{
Michael Berhanu, Adrien Guérin, and Sylvain Courrech du Pont \\ MSC, Université Paris Diderot, CNRS (UMR 7057), 75013 Paris, France \\ Fiona Raoult and Rémi Perrier \\ Université Paris Diderot, UFR de Physique, CNRS (UMR 7057), 75013 Paris, France \\ Chloé Michaut \\ Institut de Physique du Globe de Paris, Université Paris Diderot, 75013 Paris, France \\ and LGTPE, Ens Lyon, CNRS (UMR 5276), 69007 Lyon, France
}

(Received 12 October 2018; revised manuscript received 21 December 2018; published 4 April 2019)

\begin{abstract}
The uplift of an initially flat elastic membrane by an upward viscous flow is investigated experimentally. The deformed shape of the membrane results from a balance between the flow pressure, the elastic response of the membrane, and the fluid weight. This last effect becomes non-negligible for a large enough deformed area. The usual theoretical approach supposes the presence of a prewetting film regularizing the viscous stresses according to Lister et al. [Phys. Rev. Lett. 111, 154501 (2013)]. Nevertheless, in our experiments without prewetting films, the measurements are correctly described with this theory in the elastic regime. Microscale roughness of membranes could introduce an equivalent characteristic scale in the problem. An alternative explanation could be provided by the appearance of a fluid lag filled with gas, for which a new theoretical framework has been recently proposed by Ball and Neufeld [Phys. Rev. Fluids 3, 074101 (2018)]. We compare the two approaches and find that both describe reasonably our experiments. However, consistency tests of both models show that the prewetting film model is more appropriate to describe our experimental data.
\end{abstract}

DOI: 10.1103/PhysRevE.99.043102

\section{INTRODUCTION}

The physical problem of the lifting of an elastic plate by a spreading viscous flow constitutes a standard example of fluid-structure interaction in a viscous regime. However, this situation is also of strong interest in geophysics, to understand the dynamics of shallow magmatic intrusions using either analog experiments [1] or theoretical models [2]. A lift up of viscous magma penetrates between two layers of solid rocks, and the top layer is deformed by the flow [3,4]. At the crust surface, due to the balance between the pressure of the flow and the repelling force, i.e., the elastic bending and the gravity, magmatic intrusions, called laccoliths, create characteristic domes. They can be observed on Earth and other telluric bodies of the solar system [2,5-7].

Model experiments have been performed in axisymmetric geometry studying the early stage of the phenomenon [8] by injecting a fluid below an elastic membrane, where the bending elasticity opposes the flow pressure. A similar configuration has been also experimentally investigated in the case where the stretching elasticity is dominant over bending $[9,10]$. Regimes where the weight of the membrane dominates its elastic response have been reached with a different setup, by centrifugating three layers of different rheology [11]. Yet these last measurements cannot be quantitatively compared with models. Moreover, in an analog configuration where a gas is injected in a viscous fluid, the presence of an elastic membrane over the fluid has been shown to suppress fingering instabilities at the interface between fluid and gas [12-14]. In the following, we consider only the case, where a liquid is introduced between the membrane and the flat surface.

Theoretically, the propagation of the fluid below the membrane presents a major difficulty due to the singular viscous stresses at the propagating contact line $[15,16]$. At the points along the curve joining the upper membrane, the bottom substrate, and the fluid, the motion of a contact line in a viscous regime implies a divergence of the necessary stress to induce the motion. Physically the macroscopic equations become invalid at the contact line, and a microscopic model is necessary. To overcome the difficulty, most of the models of the spreading of a viscous fluid consider the existence of an initial thin prewetting film over which the injected fluid progresses. Recent experiments [8] impose such a film as a starting condition, and the measurements are in good agreement with the model where the initial thickness is a parameter. Nevertheless, the existence of a prewetting film is not justified for magmatic intrusions. This problem presents a strong analogy with the dynamic wetting problems, where the motion of an air-liquid-solid contact line during the liquid spreading is considered [17-19]. The introduction of a precursor film appears as a convenient way to introduce a cutoff length in the liquid spreading and to keep the macroscopic modeling. In the absence of a real initial prewetting film, the cutoff length could be justified by a better microscopic model of the contact line, and the main scalings could remain the same. Recently, a new set of experiments without any initial prewetting film have been performed and modeled by considering an interstice filled with vapor between the 
membrane and the fluid at the advancing front [20]. The gas pressure regularizes the divergence of the viscous stresses at the moving contact line $[16,20,21]$. This model predicts a lag distance between the front of elastic deformation and the contact line of the advancing fluid. This suggests that the spreading and growth of shallow magmatic intrusions such as laccoliths might be partly controlled by the process of gas release from the magma at the intrusion tip. In the first steps of the propagation, the same authors argue that the growth of elastic deformation is controlled by the adhesion on the substrate rather than by the viscous drag.

Here we report a set of independent experiments without prewetting film. We consider an initially flat deformable membrane of thickness $d$, which lies on a flat rigid and horizontal plate. A fluid of viscosity $v$ is injected with a flow rate $Q$ at the center of the experiment in $r=0$. Parameters are chosen to ensure a low Reynolds number flow regime. Due to the fluid spreading between the membrane and the plate, the membrane is deflected upwards. The corresponding deformation $h(r, t)$ is assumed axisymmetric (with $r$ the radial coordinate and $t$ the time) and is measured using a laser line on the top surface of the membrane. As the outer boundary of the membrane is not maintained mechanically, we assume that stretching is negligible compared to bending as long as $h \ll d$. Analysis of our results shows that our experiments are reasonably described with two models: the prewetting film (PF) model [8] and the vapor tip (VT) model [20]. As the behaviors predicted with these two approaches are quantitatively very close, we are not able to discriminate between them. The VT model could justify physically the cutoff length needed to regularize the motion of the contact line in the PF model. However, this cutoff length could be also given by the microscale roughness from the membrane and the substrate.

\section{THEORETICAL BACKGROUND}

We provide in this section the essential elements needed to describe the physical phenomenon and our measurements. Several hypotheses are needed to derive the shape and the dynamics of the deformation of the membrane by an injected fluid. We use the notations of Lister et al. [8] and of Ball et al. [20].

In the lubrication approximation, the membrane deflection and the global mass conservation are written [2]

$$
\frac{\partial h}{\partial t}=\frac{1}{12 \mu} \nabla \cdot\left[h^{3} \nabla\left(B \nabla^{4} h+\rho g h\right)\right]
$$

and

$$
Q t=2 \pi \int_{0}^{R_{F}(t)} h(r, t) r d r .
$$

$B$ denotes the bending modulus defined as $B=\frac{E d^{3}}{12\left(1-\nu^{2}\right)}$, where $E$ is the Young modulus, $v$ the Poisson ratio, and $d$ the top membrane thickness. Here $g=9.81 \mathrm{~m} \cdot \mathrm{s}^{-2}$ is the gravity acceleration, $\mu$ the fluid dynamic viscosity, and $\rho$ the fluid density. In the global mass conservation equation (2), $R_{F}(t)$ is the peeling front position, which can be assimilated in the absence of prewetting film to the location of the advancing contact line at the interface between the liquid, the rigid plate, and the deformed membrane. In the models, other quantities of interest are introduced: $h_{0}$ the initial prewetting film thickness in the PF model and $\sigma$ the pressure in the lag zone for the VT model.

The elastogravity length $L_{e g}=[B /(\rho g)]^{1 / 4}$ is the characteristic length separating the elastic regime (for $R \ll L_{e g}$ ) from the gravity regime. For a low enough flow rate, the membrane deformation is quasistatic, and the pressure field in the liquid is uniform. In the elastic regime, for a small enough film thickness, and supposing nullity of the deformation and of its gradient at the peeling front, the shape of the deformed area is written $[2,8,20]$

$$
h(r, t)=\frac{3 Q t}{\pi R_{F}^{2}(t)}\left[1-\frac{r^{2}}{R_{F}^{2}(t)}\right]^{2} .
$$

From this quasistatic shape, the curvature at the front can be expressed

$$
\kappa_{F}=\frac{24 Q t}{\pi R_{F}^{4}} .
$$

The deflection dynamics is then controlled by the advance of the peeling front $R_{F}(t)$. The two models, PF [8] and VT [20], provide two distinct solutions for the evolution of $R_{F}$ during the elastic regime.

In the PF model using a semianalytic computation, the following similarity solutions are obtained in the elastic regime [8]:

$$
R_{F}(t)=1.31\left(\frac{h_{0} B^{2} Q^{5}}{\mu^{2}}\right)^{1 / 22} t^{7 / 22}
$$

and

$$
h(0, t)=0.55\left(\frac{\mu^{2} Q^{6}}{h_{0} B^{2}}\right)^{2 / 22} t^{8 / 22} .
$$

Combining these two equations, the prewetting film thickness can be expressed as

$$
h_{0}=0.794^{22}\left(\frac{Q \mu}{B}\right)^{2} \frac{R_{F}^{8}(t)}{h^{7}(0, t)} .
$$

In the validity domain of this model, the computed value of $h_{0}$ must be constant with time to be consistent with the hypothesis of a prewetting film of thickness $h_{0}$. Moreover, from the front propagation law, another expression of the front curvature can be obtained using the peeling front velocity $\dot{R}_{F}=d R_{F} / d t[8]$ :

$$
\kappa_{F} h_{0}=1.35\left(\frac{12 \mu}{B h_{0}^{1 / 2}}\right)^{2 / 5} R_{F}^{\dot{2} / 5} .
$$

The typical size on which the front curvature is felt is given by the peeling length:

$$
L_{p, h_{0}}=\left(B h_{0}^{3} / 12 \mu \dot{R_{F}}\right)^{1 / 5} .
$$

For sufficient spreading of the fluid, when $R_{F}(t) \approx L_{e g}$, the gravity effect becomes non-negligible in Eq. (1). Lister et al. proposed solutions taking into account simultaneously 
bending and gravity [8]:

$$
R_{F}(t)=0.40 L_{e g}^{-5 / 6}\left(\frac{h_{0} B^{2} Q^{5}}{\mu^{2}}\right)^{1 / 12} t^{7 / 12}
$$

and

$$
h(0, t)=2.02 L_{e g}^{5 / 3}\left(\frac{\mu^{2} Q}{h_{0} B^{2}}\right)^{1 / 6} t^{-1 / 6} .
$$

In the VT model, in the elastic regime only $\left[R_{F}(t) \lesssim L_{e g}\right]$ the corresponding propagation solutions are found [20]:

$$
R_{F}(t)=1.52\left[\frac{B^{3} Q^{7}}{(12 \mu)^{2} \sigma}\right]^{1 / 30} t^{3 / 10}
$$

and

$$
h(0, t)=0.41\left[\frac{(12 \mu)^{2} \sigma Q^{8}}{B^{3}}\right]^{1 / 15} t^{2 / 5} .
$$

The vapor pressure in the lag zone $\sigma$ can be then deduced from these two last expressions:

$$
\sigma=(1.55)^{30}\left[\frac{B^{3}}{(12 \mu)^{2} Q^{2}}\right]^{2} \frac{h^{9}(0, t)}{R_{F}^{12}(t)} .
$$

In this VT model the front curvature is written [20]

$$
\kappa_{F \sigma} \approx\left[\frac{2^{3}(12 \mu)^{2} \sigma}{B^{3}}\right]^{1 / 7} R_{F}^{\dot{2} / 7} .
$$

The corresponding viscous peeling length is $L_{p \sigma}=$ $\left[2^{12}(12 \mu) B^{2} \dot{R}_{F} / \sigma^{3}\right]^{1 / 7}$. This last quantity is of interest, because it gives an order of magnitude of the lag length (size of the vapor cavity) and provides a condition of validity of this model consisting in $L_{p \sigma}>L_{c}$, with $L_{c}=\sqrt{B \kappa / \sigma}$, the length scale on which the adhesion curvature is felt [20]. $L_{p \sigma}<L_{c}$ corresponds to an adhesion dominant spreading controlled by interfacial adhesion [20]. Finally, $L_{p \sigma}$ could connect the two models by setting the height of liquid at the liquid front $h_{F}$ close to the tip, by the relation $h_{F}=\sigma L_{p, \sigma}^{4} /(16 B)$. Note $h_{F}$ is time dependent as the peeling length $L_{p, \sigma}$ evolves with time. We emphasize that the two models are very similar, by introducing a thickness (vertical scale) at the peeling front to regularize the stress divergence. This scale can be given by the thickness of the prewetting film $h_{0}(\mathrm{PF})$ or deduced to be equal at each time to the value of $h_{F}(\mathrm{VT})$ from the mechanical static equilibrium in the presence of a lag at the pressure $\sigma$. Nevertheless, the small-scale physics differs strongly. The regularization method provided by the VT model $[16,20]$ is original, because it proposes a macroscopic physical mechanism avoiding the divergence of the stresses, whereas the PF model, like the common regularization methods such as the slip length, introduces a microscopic cutoff length, which can be justified by a microscopic approach [18]. We note also that the effect of surface tension at the liquid-vapor interface has not been addressed theoretically in the VT model, which could affect the value of the vapor pressure found when the model is applied on experimental measurements. Finally, the same propagation method is used in both models to derive the membrane shape as a function of time. We note also that small-scale roughness of the flat substrate and of the membrane could influence the effective vertical scale at the peeling front. This question has not yet been theoretically addressed to our knowledge.

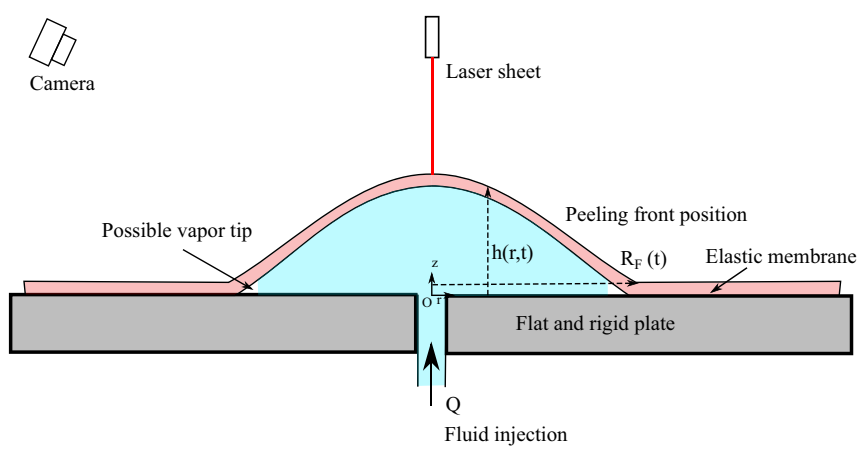

FIG. 1. Schema of the experimental setup. Glycerine-water mixture is injected with a flow rate $Q$ between an elastic membrane and a flat and rigid substrate. The resulting deformation $h(r, t)$ of the membrane is measured assuming axisymmetric geometry by imaging with a digital camera a laser line projected on the membrane top surface.

\section{EXPERIMENTAL SETUP}

The experimental device displayed in Fig. 1 is similar to the one used in Ref. [8], but without applying any initial liquid layer of known thickness, playing the role of a prewetting film. As a fluid, we use mixtures between glycerol and water. The dynamical viscosity $\mu$ varies between 0.18 and $0.82 \mathrm{~Pa} \cdot \mathrm{s}$ and the volumetric mass $\rho$ between 1234 and $1260 \mathrm{~kg} \cdot \mathrm{m}^{-3}$ at $T \approx 25^{\circ} \mathrm{C}$. The liquid is injected through an aperture in the center $(r=0)$ of diameter $4 \mathrm{~mm}$. The flow rate $Q$ of liquid injection is applied by a KD Scientific syringe pump, between $8.33 \times 10^{-8}$ and $4.5 \times 10^{-7} \mathrm{~m}^{3} \cdot \mathrm{s}^{-1}$, used with $60 \mathrm{ml} \mathrm{sy-}$ ringes. During an experiment, syringes are changed quickly in a short time $(\sim 10 \mathrm{~s})$, to ensure a nearly constant flow rate. The associated Reynolds numbers evaluated at the injection are of order $5 \times 10^{-4}$. The bottom flat and rigid plate is made of plastic (PMMA), and its dimensions are $1270 \times 1180 \mathrm{~mm}$. We have used three distinct elastic membranes of different properties:

Membrane A: Commercial rectangular silicon sheet (highconsistency silicone rubber from Sterne), $1200 \times 1000 \mathrm{~mm}$, $d=10 \mathrm{~mm}$, hardness 60 Shores A $(E=4 \mathrm{MPa}), \rho_{s} \approx$ $1400 \mathrm{~kg} \cdot \mathrm{m}^{-3}, v \approx 0.5, L_{e g}=75.5 \mathrm{~mm}$.

Membrane $B$ : Molded silicon sheet of radius approximately $250 \mathrm{~mm}, d=8 \mathrm{~mm}$, and hardness 8 Shores A ( $E=$ $0.4 \mathrm{MPa}$ ), made using Zhermack Elite 8 (addition of silicon), $\rho_{s}=1043 \mathrm{~kg} \cdot \mathrm{m}^{-3}, v \approx 0.5, L_{e g}=35.8 \mathrm{~mm}$.

Membrane $C$ : Commercial ethylene polypropylene (EPDM) sheet, $1200 \times 1200 \mathrm{~mm}, \quad d=6 \mathrm{~mm}$, hardness 70 IRHD $(E=7 \mathrm{MPa}), \quad \rho_{s}=1130 \mathrm{~kg} \cdot \mathrm{m}^{-3}, \quad v \approx 0.5$, $L_{e g}=64.5 \mathrm{~mm}$.

Small-scale roughness of membranes was also measured on small samples using a Micro-Epsilon laser profilometer. We evaluate the typical roughness by providing the root-mean square of the small-scale profile: $\sigma_{\eta}=1.92 \times 10^{-5} \mathrm{~m}$ for membrane $A, \sigma_{\eta}=4 \times 10^{-5} \mathrm{~m}$ for membrane $B$, and $\sigma_{\eta}=$ $1.63 \times 10^{-4} \mathrm{~m}$ for membrane $C$.

During the injection process, the membrane deflection is measured optically. A laser line projected on the membrane crossing the point $r=0 \mathrm{~mm}$ is imaged with a digital SLR camera (Nikon D5000, $4288 \times 4288$ pixels). The position of 


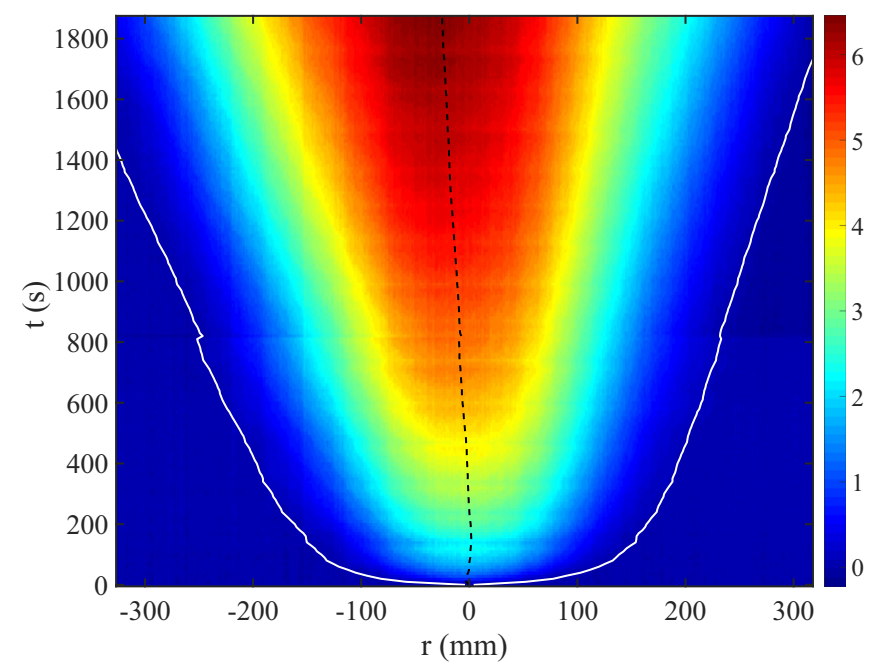

FIG. 2. Spatiotemporal diagram, the height $h$ in $\mathrm{mm}$ is depicted with a color scale and is expressed as a function of $t$ and $r$ for the measurement described in Sec. IV. Dashed black line, center of deformation, $r_{E}$. White lines, position of peeling front $R_{E}$. Membrane $A$.

the laser line is detected with a subpixel accuracy by fitting the intensity profile for each column of the image. The time lapse between two successive images is $10 \mathrm{~s}$ for most of the experiments. As the membrane is lifted up, the laser line appears then deformed on the images, and after calibration with an object of known size, $h(r, t)$ is measured along a diameter with a vertical resolution better than $0.1 \mathrm{~mm}$.

\section{MEASUREMENT PROCEDURE}

We present here in detail a specific experiment performed with membrane $A$. These measurements are in conditions close to those of previous studies $[8,20]$. The presented experiment was performed without prewetting film, for a flow rate $Q=4.50 \times 10^{-7} \mathrm{~m}^{3} \cdot \mathrm{s}^{-1}$ of a mixture of glycerol with few percent of water. The viscosity was measured with a cone plate rheometer $\mu=0.40 \mathrm{~Pa} / \mathrm{s}$, and the volumetric mass was found close to the one of pure glycerol, $\rho=1260 \mathrm{~kg} \cdot \mathrm{m}^{-3}$. The elasto-gravity length was $L_{e g}=75.5 \mathrm{~mm}$.

The deformation profile was measured on an axis every $10 \mathrm{~s}$ with the laser line technique. We display the uplift dynamic $h(r, t)$ as a spatiotemporal diagram in Fig. 2. The deformed area of the membrane grows in width and height as a function of time and reaches a height of a little more than $6 \mathrm{~mm}$. The width of this deformed area reaches a size of order a few $L_{e g}$, which means that the weight should not be negligible to predict the deflection amplitude. The end of the experiment is limited by the width of the camera window, chosen to have a sufficient spatial resolution. Four profiles are plotted in Fig. 3. The experimental profiles are satisfactorily fitted by the quasistatic-elastic solution [Eq. (3)] allowing a shift of the center $r_{E}$ :

$$
h(r)=H_{E}\left[1-\frac{\left(r-r_{E}\right)^{2}}{R_{E}^{2}}\right]^{2},
$$

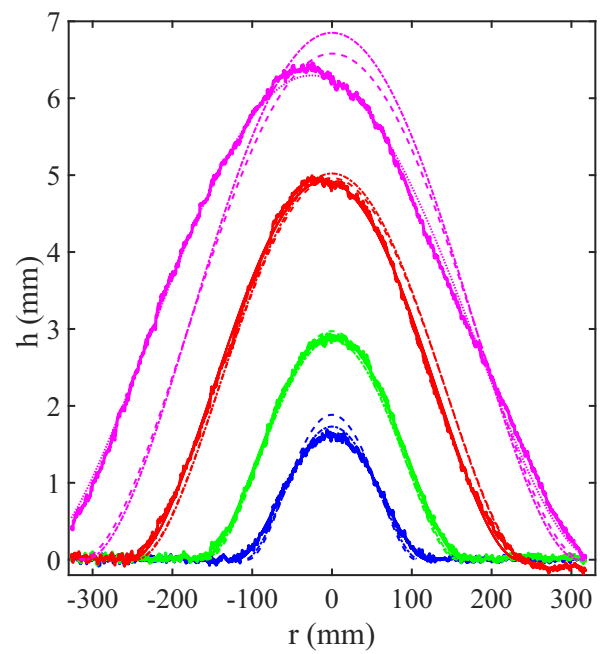

FIG. 3. Profiles of deformation $h(r)$, at $t=60 \mathrm{~s}$ (blue), $t=210 \mathrm{~s}$ (green), $t=860 \mathrm{~s}$ (red), and $t=1860 \mathrm{~s}$ (magenta) (bottom to top). Continuous lines, experimental profiles. Dotted lines, fits by Eq. (16). Theoretical solutions in pure elastic regimes from the PF model [dashed lines, Eqs. (3) and (5) using $\left.h_{0}=\left\langle h_{0 \text { eff }}\right\rangle\right]$ and from the VT model [dash-dotted lines, Eqs. (3) and (12) using $\sigma=\left\langle\sigma_{\text {eff }}\right\rangle$ ]. Membrane $A$.

defining at each time the height of deformation $H_{E}$ and the radius of deformation $R_{E}$, which is expected to be equal to the peeling front $R_{F}$. For this membrane the solution in an elastic regime appears to remain valid at least for $R_{E} \leqslant 4 L_{\text {eg }}$. Despite $H_{E}$ and the membrane thickness $d$ having the same order of magnitude, the agreement of experimental profiles with the shape predicted for pure bending suggests that the stretching is negligible in our measurements.

The agreement of the blister shape with the one predicted by Eq. (3) for a pure bending regime suggests that the injected volume $Q t$ can be measured experimentally from the measured profile by computing $\frac{\pi}{3} H_{E} R_{E}^{2}$, which is equal to $Q t$ according to Eq. (3). This last quantity is plotted as a function of time in Fig. 4 and is effectively proportional to $t$ at short time $(t<1200 \mathrm{~s})$ corresponding to the regime where gravity effects are negligible. However, the corresponding slope gives an effective flow rate $Q_{\text {eff }}=3.7 \times 10^{-7} \mathrm{~m}^{3} \cdot \mathrm{s}^{-1}$, which is $82 \%$ of the flow rate imposed by the syringe pump $Q=4.5 \times 10^{-7} \mathrm{~m}^{3} \cdot \mathrm{s}^{-1}$. The difference can be attributed to a shift between the point of maximal elevation and the injection point on which the laser line is centered, which induces a deviation from axisymmetry. This observation has been also reported in the experiments of Ball et al. [20]. Similarly, we choose in the following to take for the flow-rate value $Q_{\text {eff }}$ obtained from the temporal evolution of the measured profiles, which leads to a better agreement of experimental curves with the models.

Then we aim to compare the experimental profiles to the theoretical profiles in an elastic regime obtained by injecting the theoretical front radius $R_{F}$ from Eq. (5) (PF) or Eq. (12) (VT) into Eq. (3) for the two models. However, both models have unknown parameters: the film thickness $h_{0}$ and the gas pressure $\sigma$. We adopt the following procedure. Using, respectively, Eqs. (7) and (14) in which we inject the 


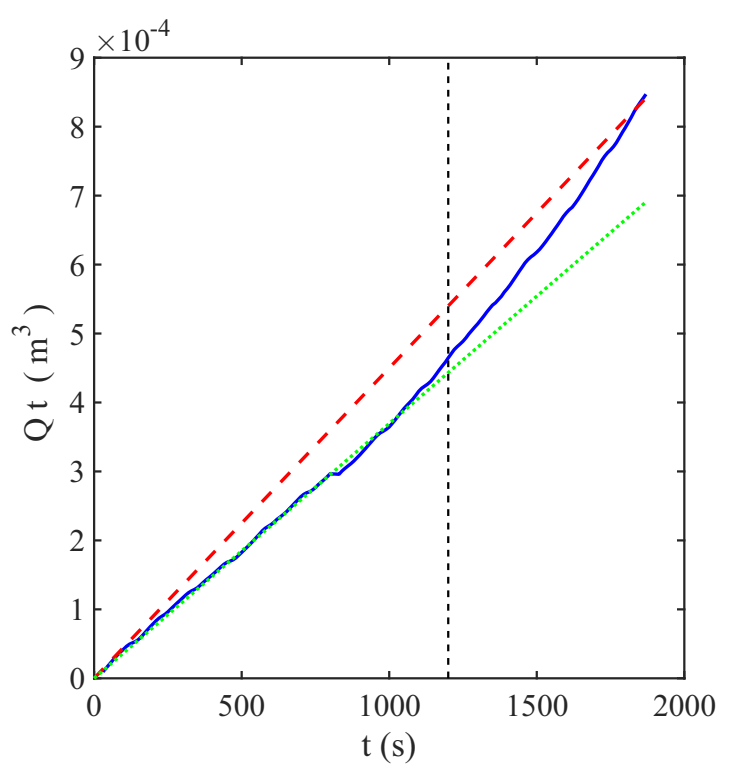

FIG. 4. Injected volume, i.e., the flow rate multiplied by the time as a function of time. Red dashed line, $Q t$ product of the flow rate $Q$ imposed by the syringe pump by the time $t$. Blue continuous line, experimental estimation $Q t=\frac{\pi}{3} H_{E} R_{E}^{2}$, valid at short time, when gravity effects are negligible according to the theoretical profile Eq. (3). Vertical dash line, end of the elastic regime: for $t<t \approx$ $1200 \mathrm{~s} \frac{\pi}{3} H_{E} R_{E}^{2}$ is proportional to $t$. Green dotted line, linear fit of the previous curve, providing the effective flow rate $Q_{\text {eff }}$ used to describe the experiments in the following. We note that $Q_{\text {eff }}<Q$.

fitted profile parameters $H_{E}$ and $R_{E}$, we plot the expected value of $h_{0}$ and $\sigma$ as a function of time in Fig. 5. If the models are valid, both values must be constant with time. Experimentally, we observe that the effective values of $h_{0}$
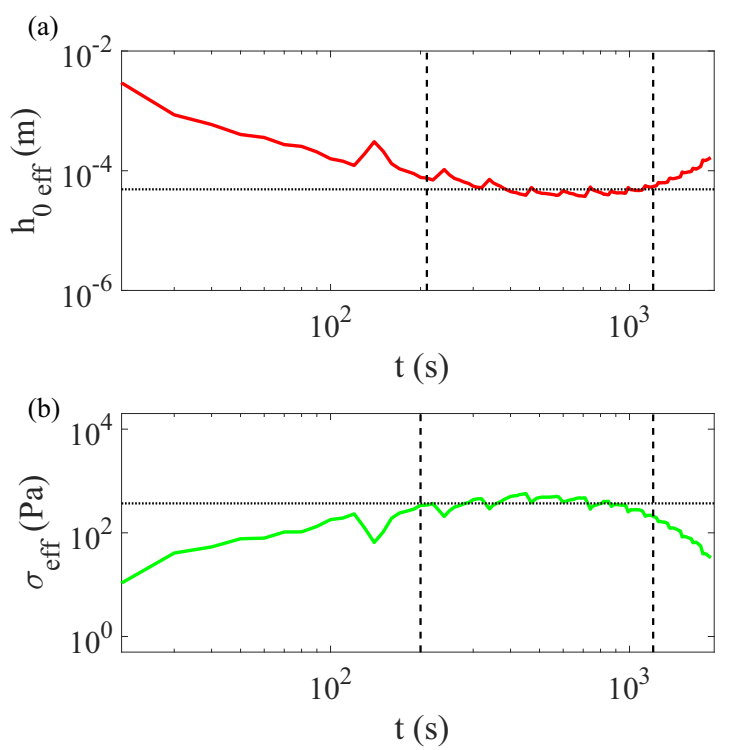

FIG. 5. (a) Effective prewetting film thickness using Eq. (7). For $t \in[210,1200] \mathrm{s}, h_{0 \text { eff }}$ is nearly constant, and we find $h_{0}=$ $\left\langle h_{0 \text { eff }}(t)\right\rangle=4.89 \times 10^{-5} \mathrm{~m}$. (b) Effective vapor tip pressure using Eq. (14). For $t \in[200,1200] \mathrm{s}, \sigma_{\text {eff }}$ is nearly constant, and we find $\sigma=\left\langle\sigma_{\text {eff }}(t)\right\rangle=370 \mathrm{~Pa}$. Membrane $A$. and $\sigma$ are roughly constant on the interval [200, 1200] s. The noticeable oscillations are attributed to a defect in injection when a new syringe replaces the empty one in the syringe pump. At long times, the gravity contribution becomes significant. On the intermediate interval, the average values of effective parameters give $h_{0}=\left\langle h_{0 \text { eff }}\right\rangle=4.89 \times 10^{-5} \mathrm{~m}$ and $\sigma=\left\langle\sigma_{\text {eff }}\right\rangle=370 \mathrm{~Pa}$. The effective values are not exactly constant with time, and they are obtained from large powers of measured quantities. These average values are thus considered as an approximate estimation of the unknown parameters providing the right order of magnitude. This last value of the pressure is found to be considerably smaller than the one of $101 \times 10^{3} \mathrm{~Pa}$ in Ref. [20]. However, the power of $\sigma 1 / 30$ in Eq. (12) and 1/15 in Eq. (13) induces a strong uncertainty. Physically, the order of magnitude of the water partial pressure at thermodynamic equilibrium for a $90 \%-10 \%$ mixture of glycerol-water at $25^{\circ} \mathrm{C}$ is of order $700 \mathrm{~Pa}$ [22], which has the same order of magnitude than our experimental value. A void cavity should be quickly filled with water vapor at a pressure of a few hundreds Pa. Concerning the PF model, as experiments are performed without initial prewetting film, we assume that the effective thickness $h_{0 \text { eff }}$ found by this procedure corresponds qualitatively to the cutoff length scale needed to regularize the lubrication problem. We observe also that $\left\langle h_{0 \text { eff }}\right\rangle=4.89 \times 10^{-5} \mathrm{~m}$ is of order of the membrane roughness $\sigma_{\eta}=1.92 \times 10^{-5} \mathrm{~m}$.

These values of $h_{0 \text { eff }}$ and $\sigma_{\text {eff }}$ are then used to obtain the theoretical profiles in Fig. 3. Both models give close predictions and cannot be discriminated from the measurement. Except for the last profile at $t=1860 \mathrm{~s}$, which does not belong to the pure bending regime, the experimental profiles are well reproduced by the theoretical profiles for the prewetting film model and the vapor tip model, when we estimate the flow rate as $Q_{\text {eff }}$. We observe also by comparing to other experimental runs a significant sensitivity to initial conditions, inducing a substantial shift between the maximum of deformation and the center of the experiment. This shift of order $5 \%$ of $R_{E}$ (less than $12 \mathrm{~mm}$ ) does not appear in a particular side. A similar statement has been reported in the experiments in Ref. [20], where the maximum of deformation was systematically off centered from the injection point, resulting in profiles smaller than the analytic solutions. At long times, the deformation seems to lose its axisymmetric shape, making the reconstruction more questionable.

To better compare with the temporal scalings in elastic regimes given by Eqs. (5) and (12), we use the height $H_{E}$ and the radius $R_{E}$ extracted from experimental profiles. During the earliest times the top deformation is not well detected, therefore the first four values are not displayed (typically $t \leqslant 40 \mathrm{~s}$ ). At long times, corresponding to the regimes where gravity is no longer negligible, these estimations become more questionable. Therefore, we have also fitted the profiles by a Gaussian function:

$$
h(r) \sim H_{G} \exp \left[\frac{-\left(r-r_{G}\right)^{2}}{2 R_{G}^{2}}\right] .
$$

We found that $R_{E} \approx 2.5 R_{G}$ in the elastic regime, then $R_{E} \lesssim$ $2.5 R_{G}$ at longer time, in agreement with a flow developing steeper slopes as gravity becomes more important [2]. A last estimation of $R_{F}$ is obtained from the experimental profiles. 


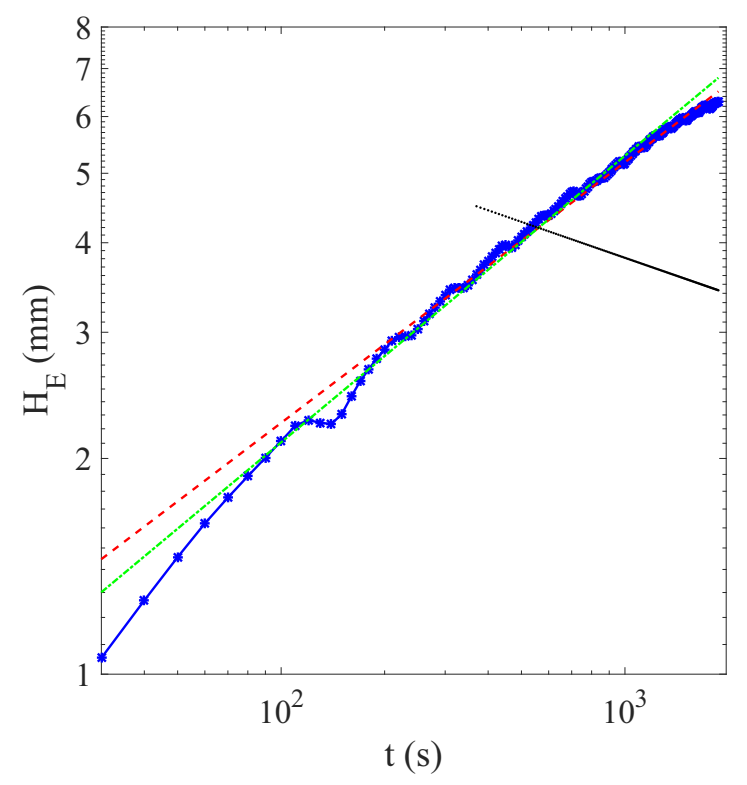

FIG. 6. Height of deformation $H_{E}$ (blue continuous line with asterisks) as a function of time. The dashed red line is the elastic solution (6) for the PF model, the dash-dotted green line is the elastic solution (13) for the VT model, and the dotted line is the gravity-elastic solution (11). Membrane $A$.

The maximal deformation is extracted $H_{S}$, and we define the radius $R_{1}$ by $h\left(r=R_{1}\right)=0.1 H_{S}$. Then we find $R_{E} \approx$ $1.21 R_{1}$ during the entire experiment. $R_{E}$ appears thus to be an acceptable estimation of $R_{F}$ even outside the elastic regime. The experimental height is plotted as a function of time in Fig. 6. In the elastic regime the two theoretical models provide very close curves. For $t \in[200,1200] \mathrm{s}, H_{E}$ follows well the propagation scaling given by the elastic solution $\left(t^{8 / 22}\right.$ for the PF model or $t^{2 / 5}$ for the VT model).

Then the equivalent radii $R_{E}$ are plotted as a function of time $t$ in Fig. 7. Similarly for $1<R / L_{e g}<3$, the elastic scaling is well verified. The measured values of $R_{E}(t)$ follow the elastic scalings $\left(t^{7 / 22}\right.$ for the PF model or $t^{3 / 10}$ for the VT model). At longer time, $R_{E}$ grows faster, while the effective prewetting film thickness and vapor pressure values largely vary with time. This fact could be explained by the beginning of the gravity-elastic regime. The profile departs indeed from the bell shape given by Eq. (3) to adopt a flatter and wider shape. However, despite the large scale of the membrane, the regime where gravity influences the deformation is not reached as the expected decrease of $H_{E}$ with time is not observed.

In order to evaluate the peeling length and thus discuss the validity of models, it is necessary to estimate the propagation front velocity $\dot{R}_{F}$. An estimation is obtained by numerically differentiating $R_{E}(t)$ after smoothing the curve during $40 \mathrm{~s}$. Surprisingly, we observe in Fig. 8 after an initial decrease a nearly constant velocity in the range where the elastic regime is verified. The order of magnitude of the measured velocity is compatible with the slow velocity decrease predicted theoretically. From this curve we obtain an order of magnitude of the front velocity $c \approx 0.117 \mathrm{~mm} / \mathrm{s}$, and we deduce the corresponding peeling lengths $L_{p h_{0}} \approx 9.6 \mathrm{~mm}$ and $L_{p \sigma} \approx 69 \mathrm{~mm}$.

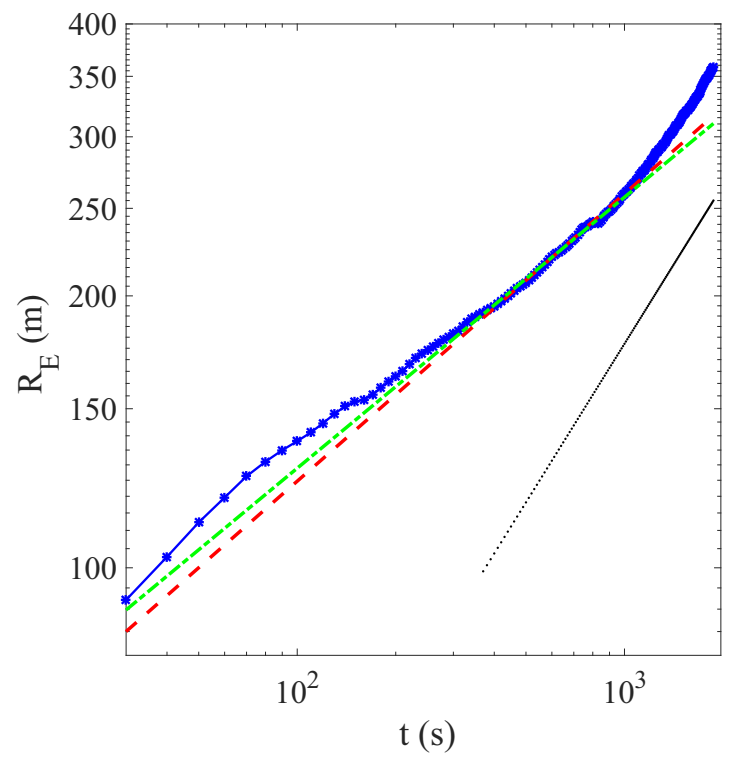

FIG. 7. Radius of deformation $R_{E}$ (blue continuous line with asterisks) as a function of time. The dashed red line is the elastic solution (5) for the PF model, the dash dot green line is the elastic solution (12) for the VT model, and the dot line is the gravity-elastic solution (10). Membrane $A$.

This last value appears too large, as the lag observed from below the PMMA plate is not clearly visible with the naked eye and should be of order of one millimeter.

Then we estimate the front curvature $\kappa_{F}$ using Eq. (4), which is valid when the quasistatic elastic shape is verified. The result is plotted versus time in Fig. 9 and compared to the two predictions from elastic models [Eq. (8) for the PF model and Eq. (15) for the VT model]. To check the consistency of

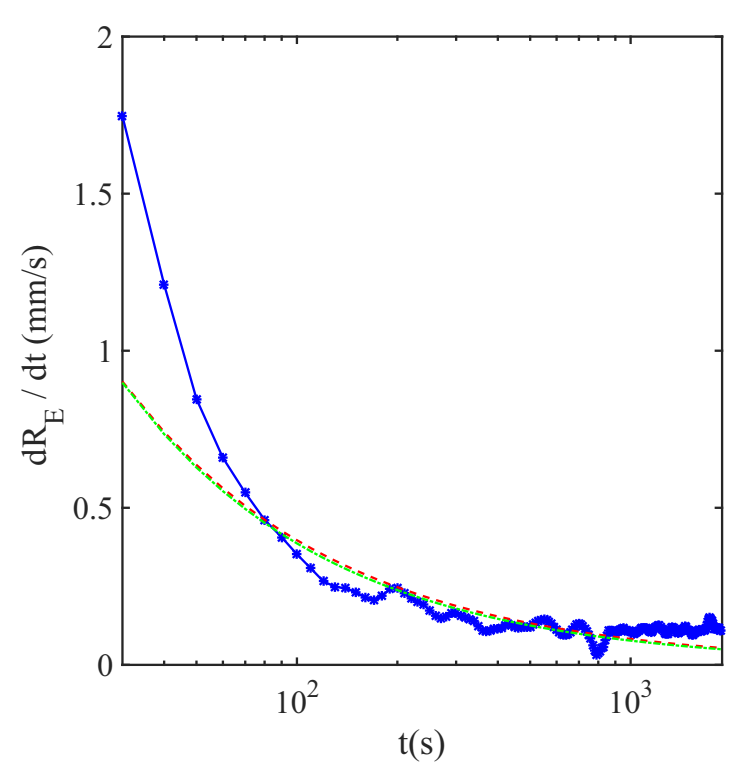

FIG. 8. Blue plain line with asterisks, front velocity $d R_{E} / d t$ versus time. The dashed red line is the elastic solution (5) for the PF model; the dash-dotted green line is the elastic solution (12) for the VT model. Membrane $A$. 


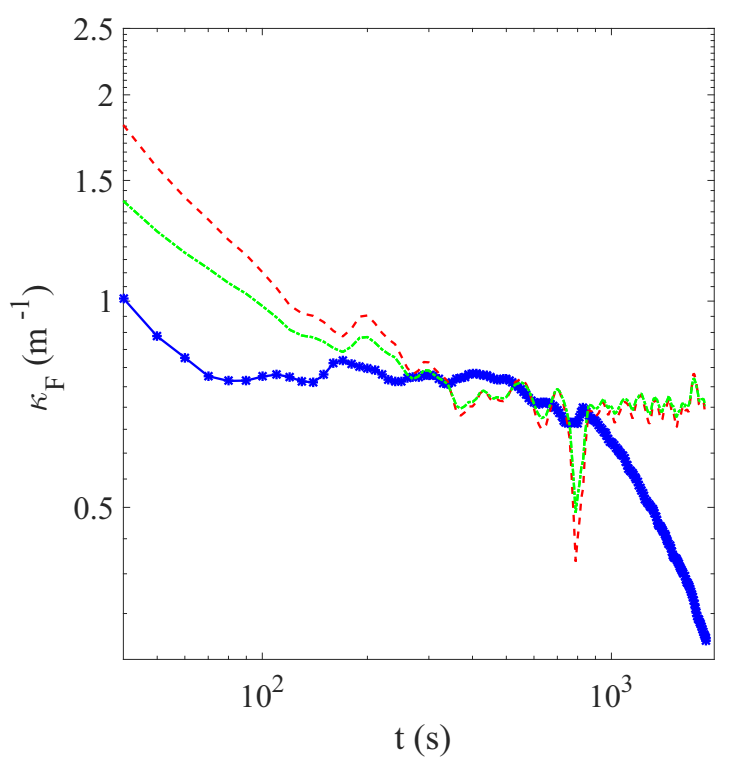

FIG. 9. Blue plain line with asterisks, front curvature $\kappa_{F}$ estimated using Eq. (4) versus time, valid in the elastic regime. The dashed red line is the curvature estimation from Eq. (8) for the PF model, using the experimental front velocity $\dot{R}_{F}$. The dash-dotted green line is the curvature estimation from Eq. (15) for the VT model. Membrane $A$.

the models, we compare these results to Eqs. (8) and (15), where the experimental values of the front velocity have been injected, rather than the propagation expressions. The consequence is a saturation of the curvature instead of a slow decrease in $t^{-3 / 11}$ or $t^{-1 / 5}$. In the domain $t \in[200,1200] \mathrm{s}$, the curvature flattens around $1 \mathrm{~m}^{-1}$ before decreasing. From our experimental values of $\kappa_{F}$, we find $L_{c}=\sqrt{B \kappa_{F} / \sigma} \approx 28 \mathrm{~mm}$, which is smaller than the peeling length (with lag hypothesis) $L_{p \sigma} \approx 69 \mathrm{~mm}$, but has the same order of magnitude. We have thus $L_{p \sigma}>L_{c}$, which ensures that our experiments are in a viscous dominant spreading [20]. However, due to the lack of scale separation, the adhesion might remain non-negligible in the front propagation. Yet the energy of adhesion is difficult to estimate. As we do not use an adhesive mount film like in Ref. [20], we expect to have a significantly smaller value than their estimation of energy of adhesion $\Delta \gamma \approx 0.85 \mathrm{~J} \cdot \mathrm{m}^{-2}$.
Finally, the typical film height given by the VT model $h_{F}=$ $\sigma L_{p, \sigma}^{4} /(16 B)$ is found equal to $h_{F} \approx 1.28 \mathrm{~mm}$, which is one order of magnitude larger than the effective film thickness $h_{0 \text { eff. }}$.

The same data processing has been performed with eight other measurements. We find systematically a smaller effective flow rate $Q_{\text {eff }}$, with $Q_{\text {eff }} \approx 0.7 Q$. The experiments with membrane $C$ (more rigid and thinner) give similar results to those presented in this section for membrane $A$. In contrast, the measurements performed with the membrane $B$, which is smoother and of smaller dimensions, provide qualitatively different results. The smaller Young modulus leads to a two times smaller elastic-gravity length $L_{e g}=35.8 \mathrm{~mm}$. However, the smaller size seems to introduce significant boundary effects, like an uplift at the border and a localized fluid leakage at one border. The shift between the maximum of the deformed area and the injection point is about $30 \%$ of $R_{E}$ and larger than with membrane $A$ but remains smaller than $L_{e g}$. We find again a region where the elastic regime is clear, with its characteristic shape and thickness-radius evolution, though we cannot discriminate between the two models. In the elastic regime, these experiments do not also discriminate the PF and the VT model. Due to the smaller $L_{e g}$, the effects of weight become non-negligible when $R_{E}$ reaches approximately $4 L_{e g}$. The height starts to saturate, the profile becomes wider, and the agreement with the quasistatic elastic solution decreases. However, the intermediate theoretical solution [Eq. (11)] does not provide the correct magnitude. The scalings are tested on range that is too short to test the power law $\left(R \sim t^{7 / 12}\right.$ and $h \sim t^{-1 / 6}$ ) in this intermediate regime. To be able to test these regimes, larger membranes with a similar value of the bending modulus are needed, but these membranes are harder to mold homogeneously.

\section{SYNTHESIS}

In order to compare the measurements in the elastic regime, we consider nine different experiments realized with the three membranes and various parameters (Table I), without initial prewetting film. As we do not observe notable differences between the profiles predicted by the two models (PF and VT) in the elastic regime, we rescale the data by the characteristic scales provided by the PF model $[2,8]$. Radial coordinates are then rescaled by the elastogravity length $L_{e g}=[B /(\rho g)]^{1 / 4}$.

TABLE I. Summary of parameters for each measurement. The dimensionless thickness $\epsilon$ is obtained by finding experimentally the effective $h_{0}$ [Eq. (7)]. The peeling length $L_{p, h_{0}}$ is evaluated using Eq. (9). Characteristic time $\tau^{*}$ and height $h^{*}$ are defined when $R_{E}=2 L_{e g}$, corresponding roughly to the middle of the elastic regime. The effective lag pressure $\sigma$ is found experimentally using Eq. (14).

\begin{tabular}{|c|c|c|c|c|c|c|c|c|c|}
\hline & $L_{e g}(\mathrm{~mm})$ & $L_{H}(\mathrm{~mm})$ & $\mathrm{T}(\mathrm{s})$ & $h_{0}(\mathrm{~mm})$ & $\epsilon$ & $L_{p, h_{0}}(\mathrm{~mm})$ & $\tau^{*}$ & $h^{*}$ & $\sigma(\mathrm{Pa})$ \\
\hline$A$ run 1 & 75.5 & 2.60 & 126 & 0.049 & 0.019 & 9.6 & 1.1 & 0.86 & 370 \\
\hline$A$ run 2 & 75.5 & 2.41 & 158 & 0.023 & 0.0099 & 7.7 & 1.1 & 0.97 & 1200 \\
\hline$A$ run 3 & 75.5 & 2.54 & 136 & 0.098 & 0.038 & 13 & 1.6 & 1.2 & 490 \\
\hline$B$ run 4 & 35.8 & 2.03 & 27.0 & 0.013 & 0.0063 & 2.7 & 2.6 & 1.8 & 5700 \\
\hline$B$ run 5 & 35.8 & 1.82 & 82.0 & 0.0049 & 0.0027 & 1.5 & 3.0 & 2.0 & 14000 \\
\hline$B$ run 6 & 35.8 & 1.70 & 101 & 0.014 & 0.0080 & 2.9 & 3.1 & 1.7 & 3400 \\
\hline$B$ run 7 & 35.8 & 2.09 & 24.7 & 0.056 & 0.027 & 6.3 & 2.8 & 1.5 & 1050 \\
\hline$C$ run 8 & 64.5 & 2.34 & 126 & 0.95 & 0.41 & 53 & 0.71 & 0.67 & 4.8 \\
\hline$C$ run 9 & 64.5 & 2.50 & 104 & 0.76 & 0.31 & 45 & 0.96 & 0.62 & 15 \\
\hline
\end{tabular}



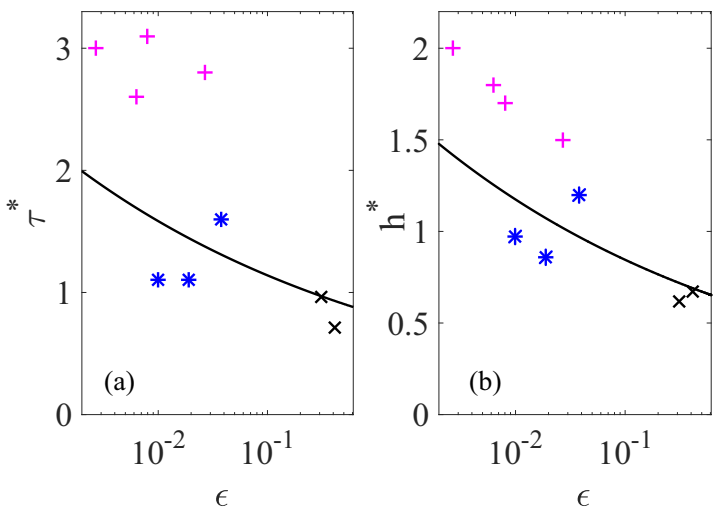

FIG. 10. Dimensionless time $\tau^{*}=t^{*} / T$ (a) and height $h^{*}=$ $h_{E}^{*} / H$ (b) corresponding to $R_{E} / L_{e g}=2$. Blue $*$, membrane $A$; magenta + , membrane $B$; black $\times$, membrane $C$; continuous black line, theoretical scaling (5) and (6) with PF model.

The typical height $L_{H}=[12 Q \mu /(\pi \rho g)]^{1 / 4}$ scales the vertical deformation, and the typical time $T=\left(\pi L_{H} L_{e g}^{2}\right) / Q$ scales the time. The thickness of the prewetting film is made dimensionless by introducing $\epsilon=h_{0} / L_{H}$. The effective value of $h_{0}$ is computed from the experimental data in the elastic regime, using the method described previously. Run 1 with membrane $A$ has been presented in detail in Sec. IV. Estimated values of $\sigma$ for the VT model are indicated and strongly vary between measurements with the different membranes, which appears difficult to justify physically. On the other hand, the characteristic film height $h_{0}=\epsilon L_{H}$ is of order $5 \times 10^{-5} \mathrm{~m}$ for membrane $A, 1 \times 10^{-5} \mathrm{~m}$ for membrane $B$, and $9 \times 10^{-4} \mathrm{~m}$ for membrane $C$, which, for the first two values at least, is of the same order of the estimated roughness of the membranes. This constitutes an additional reason to describe our measurements with the PF model. For membrane $C$, it appears that its larger roughness and its grid texture induce a large effective prewetting film of order of a millimeter. To compare each experiment we define the specific time $\tau^{*}=t / T$ and height $h^{*}=h_{E} / H$, for which $R_{E} / L_{e g}=2$. In agreement with numerical simulations with small prewetting film thicknesses [23], this radius of front propagation corresponds to an elastic regime, despite $R_{E} / L_{e g}$ is of order one.

In order to test graphically the agreement of the PF models with the data for all measurements, we plot in Fig. $10 \tau^{*}$ and $h^{*}$ as a function of the dimensionless prewetting film thickness $\epsilon$. The theoretical description of the PF model provides the expected first-order variations for all membrane (Fig. 10), in particular regarding the evolution of $h^{*}$ with $\epsilon$ (Fig. 10, right). However, we observe a scattering of the points and remark that the nature of the membranes defines three different families of points. The fit is better for membrane $A$ and $C$, while the values of $\tau^{*}$ and $h^{*}$ obtained with membrane $B$ are significantly larger than expected significantly, suggesting that the membrane is easier to bend. Stretching should be more important for this smoother membrane, because the typical deformation is close to the membrane thickness.

Then, always with the rescaling from the PF model, we compare the measurements with the numerical simulations of the problem in the framework of the PF model considering
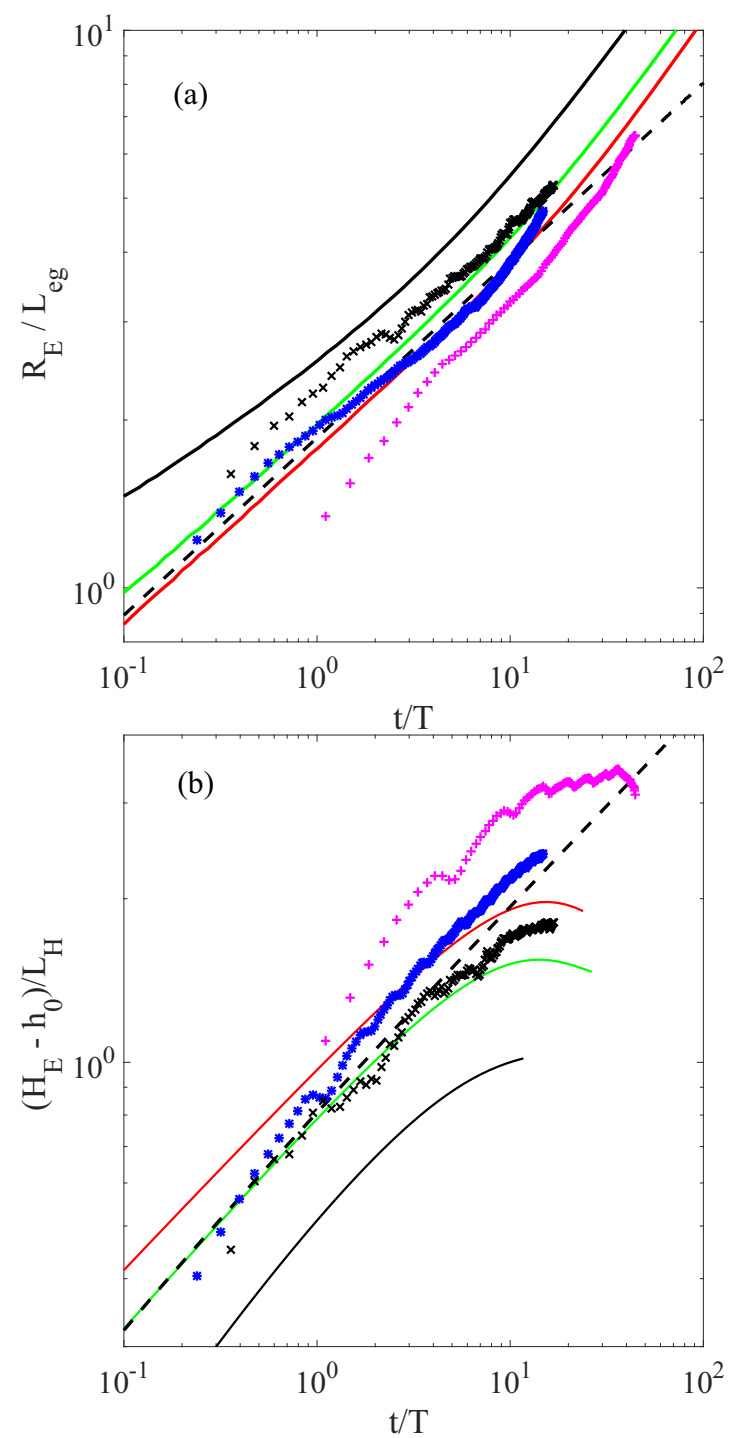

FIG. 11. Comparisons of experimental results with numerical simulations as a function of rescaled time $t / T$. (a) Rescaled front radius $R_{E} / L_{e g}\left(R_{F} / L_{e g}\right.$ in numerical simulations, $R_{E} / L_{e g}$ in experiments). (b) Top deformation $h(r=0, t) / L_{H}\left(H_{E} / L_{H}\right.$ in experiments). Plain lines are numerical simulations displayed for three dimensionless thicknesses $\epsilon$ [from bottom to top in (a) $\epsilon=0.01, \epsilon=0.05$, and $\epsilon=0.3$ and ordered inversely in (b)]. Dashed line theoretical scaling Eqs. (5) and (6) with $\epsilon=0.05$. Blue $*$, membrane $A$ run 1 (measurement described in Sec. IV); magenta + , membrane $B$ run 4 ; black $\times$, membrane $C$ run 8 .

bending and gravity. The details of the numerical algorithms can be found in the Ph.D. thesis of Thorey [24], and the corresponding results are presented in Thorey et al. [23]. In Fig. 11 we plot as a function of $t / T$ the front radius (a) $R_{E} / L_{e g}$ and central height of deformation (b) $\left(H_{E}-\right.$ $\left.h_{0}\right) / L_{H}$ for each membrane $A, B$, and $C$ corresponding, respectively, to values of $\epsilon=0.019$ (run 1), $\epsilon=0.0063$ (run 4 ), and $\epsilon=0.41$ (run 8). For comparison results of numerical simulations are plotted for $\epsilon=0.01, \epsilon=0.05$, and $\epsilon=0.3$ in the same graph. Simulations provide exactly the position of the peeling front $R_{F}$ and the top deformation $h(r=0)$ in 
dimensionless units without fitting the profiles as in experiments. The expected trends are well observed: the power laws in time from the PF model are fairly followed, as long as $R_{E} \lesssim$ $4 L_{\text {eg }}$, i.e., the influence of gravity is negligible. An increase of $\epsilon$ induces a larger front radius $R_{E}$ and a smaller height of deformation $H_{E}$. However, the experiments and the numerical simulations do not perfectly collapse. In particular, for these parameter values, the beginning of the gravity regime should be observable in the experiments. A saturation of the height is visible for the membrane $B$, but it is observed at a larger value of $\left(H_{E}-h_{0}\right) / L_{H}$. The limited size of the membranes and possible departure from the axisymmetry could explain some differences with the simulations. Moreover, the adhesion effects do not exist with a prewetting film. Although adhesion at the tip is not dominant in the experiments (the temporal scaling would be modified), its effects could constitute an other source of difference between the experimental and numerical results. In Ref. [8], the experiments performed with a prewetting film are in better agreement with numerical solutions of PF model, but the experimental results are presented for only one membrane. In our experiments, the effective dimensionless thickness of the prewetting film $\epsilon$ presents a larger range of variation than in Ref. [8].

\section{CONCLUSION}

An experimental study of the deformation of an elastic membrane by a viscous flow has been performed. This experiment is motivated for a better understanding of the physical mechanisms at play in the dynamics of magmatic intrusions in the upper crust. Following Ref. [16], the prewetting film (PF) and the vapor tip (VT) models are two theoretical methods to regularize the singular dynamics at the contact line. Whereas the precise small-scale physics and the scaling exponents differ, the predicted large-scale behavior appear very similar with both models, which are obtained with the same theoretical method, balancing membrane elasticity and lubrication pressure in the fluid. We performed several experiments with three different membranes without applying any initial prewetting film contrary to Ref. [8]. Despite the absence of a prewetting film in our experiments, the PF model describes well our results when $R_{E}<4 L_{\text {eg }}$ and gravity effects are negligible. An effective thickness of a virtual film $h_{0}=$ $\left\langle h_{0 \text { eff }}\right\rangle$ can be deduced from the measurements and provides a consistent order of magnitude $h_{0} \sim 10^{-4} \mathrm{~m}$. This length can be interpreted as the small cutoff length scale needed to regularize the viscous stresses for an advancing wetting front. Quantitatively, the agreement with the model is less convincing mainly for the smoother membrane $B$ and could be due to the finite size of the membrane, the adhesion on the substrate, and possible stretching effects. It would be interesting to quantify the adhesion energy and to incorporate it in the models in combination with elasticity. Recently, Ball et al. [20] carried out similar experiments without prewetting films, but with a controlled adhesion strength using adhesive bonding on the bottom substrate. In their study, adhesion dominant spreading has been found relevant for fluids of moderate viscosity, whereas for viscosities comparable to those of the fluids used in in this study, the VT model has been used to describe their data. We have also analyzed our measurements regarding the VT model, and we also found a qualitative agreement. As the predictions of both models are very close, it is not possible to differentiate them. We note that the VT model introduces also an effective scale for the film thickness $h_{0}$ by $h_{F}=\sigma L_{p, \sigma}^{4} /(16 B)$ (see the end of Sec. II). Nevertheless, the order of magnitude of the effective pressure in the gas cavity (about $500 \mathrm{~Pa}$ for membrane $A$, $5000 \mathrm{~Pa}$ for membrane $B$, and $10 \mathrm{~Pa}$ for membrane $C$ ) is more difficult to justify by physical arguments. Moreover, we estimate for the membrane $A$ a peeling length $L_{p \sigma}$ of order $70 \mathrm{~mm}$, which appears too large compared to the expected lag length. Therefore, we give more credence to the PF model to describe our experimental data, whereas no initial prewetting film of known thickness has been applied.

The microscale roughness of membranes could be indeed another possible mechanism introducing a characteristic length scale in the spreading problem. We observe that the typical roughness for each membrane provides the order of magnitude of the effective thickness of the virtual prewetting film, except for membrane $C$ (which has a periodic texture).

Finally, we notice also a strong sensitivity of experiments to initial conditions and a tendency to evolve to a nonaxisymmetric shape. Even with larger soft membranes, it becomes experimentally difficult to observe clearly the intermediate regime combining elasticity and gravity. A three-dimensional reconstruction of the membrane shape along time (for example, by measuring the deformation of projected fringes and deducing the elevation using the Fourier transform Profilometry method [25]) combined with a bottom accurate imaging of the liquid spreading [20] make it appear necessary to go further with these studies.

\section{ACKNOWLEDGMENTS}

The project was initiated during an undergraduate experimental project, under the supervision of Michael Berhanu, Sylvain Courrech du Pont, Julien Derr, Marc Durand, José Ocariz, and Cécile Roucelle. We thank Kamar Sarah Jarraya and Gabriel Le Goff for participating in the preliminary experiments. We thank also Wladimir Toutain and Michel Laurent for technical assistance and for lending parts of the experimental device. Finally, we acknowledge Adrian Daerr for discussions.
[1] J. L. Kavanagh, T. Menand, and R. S. J. Sparks, Earth Planet. Sci. Lett. 245, 799 (2006).

[2] C. Michaut, J. Geophys. Res. (Solid Earth) 116, B05205 (2011).
[3] A. M. Johnson and D. D. Pollard, Tectonophysics 18, 261 (1973).

[4] D. D. Pollard and A. M. Johnson, Tectonophysics 18, 311 (1973). 
[5] C. Michaut, D. Baratoux, and C. Thorey, Icarus 225, 602 (2013).

[6] C. Michaut and M. Manga, J. Geophys. Res. Planets 119, 550 (2014).

[7] M. Manga and C. Michaut, Icarus 286, 261 (2017).

[8] J. R. Lister, G. G. Peng, and J. A. Neufeld, Phys. Rev. Lett. 111, 154501 (2013).

[9] D. Pihler-Puzovic, A. Juel, G. G. Peng, J. R. Lister, and M. Heil, J. Fluid Mech. 784, 487 (2015).

[10] G. G. Peng, D. Pihler-Puzovic, A. Juel, M. Heil, and J. R. Lister, J. Fluid Mech. 784, 512 (2015).

[11] J. M. Dixon and D. G. Simpson, J. Struct. Geol. 9, 87 (1987).

[12] D. Pihler-Puzovic, P. Illien, M. Heil, and A. Juel, Phys. Rev. Lett. 108, 074502 (2012).

[13] T. T. Al-Housseiny, I. C. Christov, and H. A. Stone, Phys. Rev. Lett. 111, 034502 (2013).

[14] A. Juel, D. Pihler-Puzovic, and M. Heil, Annu. Rev. Fluid Mech. 50, 691 (2018).

[15] J. Flitton and J. King, Euro. J. Appl. Math. 15, 713 (2004).
[16] I. Hewitt, N. Balmforth, and J. D. Bruyn, Eur. J. Appl. Math. 26, 1 (2015).

[17] L. H. Tanner, J. Phys. D 12, 1473 (1979).

[18] D. Bonn, J. Eggers, J. Indekeu, J. Meunier, and E. Rolley, Rev. Mod. Phys. 81, 739 (2009).

[19] J. H.Snoeijer and B. Andreotti, Annu. Rev. Fluid Mech. 45, 269 (2013).

[20] T. V. Ball and J. A. Neufeld, Phys. Rev. Fluids 3, 074101 (2018).

[21] Z.-Q. Wand and E. Detournay, J. Appl. Mech. 85, 041010 (2018).

[22] Glycerine Producers' Association, Physical Properties of Glycerine and Its Solutions (Glycerine Producers' Association, New York, 1963).

[23] C. Thorey and C. Michaut, J. Fluid Mech. 805, 88 (2016).

[24] C. Thorey, Dynamics of shallow magmatic intrusions: Theory and application to the detection of planetary intrusion, Ph.D. thesis, Université Paris Diderot (2015).

[25] P. Cobelli, A. Maurel, V. Pagneux, and P. Petitjeans, Exp. Fluids 46, 1037 (2009). 